\title{
Random Reliability Analysis of Gravity Retaining Wall Structural System
}

\author{
Zun-Qun XIAO ${ }^{1,2,3, a, ~}{ }^{*}$, Jie HUANG ${ }^{1, b}$, Ya-Jun WANG ${ }^{1, c}$, Cai-Yun XU ${ }^{4, d}$, \\ Huan XIA $\mathrm{A}^{5, \mathrm{e}}$
}

${ }^{1}$ School of Resource and Civil Engineering in Wuhan Institute of Technology, Wuhan 430000 China

${ }^{2}$ School of Civil Engineering and Mechanics in Huzhong University of Science and technology, Wuhan, 430000 China

${ }^{3}$ China Petroleum Pipeline Scientific Research Institute, Langfang, 065000 China

${ }^{4}$ Changjiang River Scientific Research Institute, Wuhan, 430014 China

${ }^{5}$ Guizhou University of Finance and Economics in Guiyang 550004 China

a, “xiaozunqun@126.com, bHangjie@163.com, 'wangyajun@163.com, 'xucaiyun860920@163.com, exh240@126.com

${ }^{*}$ Corresponding author

Keywords: FOSM, Fuzzy Random Reliability, $\lambda$ Cut Set, Interval Numbers, Retaining Wall.

\begin{abstract}
According to present deficiency of gravity retaining wall design method and considering the fuzzy uncertainties of the parameters of stochastic variables, first-order second-moment method (FOSM) is adopted to set up fuzzy random reliability analyzing model. By using decomposing theorem and by introducing $\lambda$ cut set, fuzzy numbers were convened into a series of interval numbers and fuzzy random reliability computing technology was received. The major instability modes of gravity retaining walls are overturning and sliding. Two structural performance functions against overturning and sliding of gravity retaining walls were established and the fuzzy random variables were confirmed. Two kinds of invalid modes are calculated separately by the use of fuzzy random reliability computation method, which two fuzzy random reliability indexes and corresponding failure probabilities are obtained. Comparing the result of computation method with that of related literature, it is shows that obvious differences are between them. It is pointed out that structural performance function $\mathrm{Z}$ of the gravity retaining wall is assumed as normal distribution in related literature, which contributing to errors in the probability density function $f(Z)$ in this treatment. The calculated results of fuzzy random reliability by adopting $\lambda$ cut set way are conformed to reality better.
\end{abstract}

\section{Introduction}

The traditional design of retaining wall adopts the definite value method; using safety coefficient signifies stability of the retaining wall structure. But random uncertainty and fuzzy uncertainty has been neglected while analyzing stability of retaining wall by the design method of definite value. Random uncertainty mostly refers to that randomness of every soil physics mechanics index causes randomness of the course of loosing steady of retaining wall .Fuzzy uncertainty mainly means there exist a fuzzy transition region of retaining wall between unstable and stable. As to introducing reliability theory to design calculation of retaining wall, some domestic and international scholars have already made certain work. Basing on probability, Enrique Castillo combined the traditional safety coefficient method and reliability method together and puts forward a new retaining wall design. [1] Zhang jian-Ren utilized central point method of FOSM to calculate some retaining wall, and carried on the sensitiveness analysis of the parameter [2]. Wang Liang etc. used central point method of FOSM to calculate resisting slippery reliability of road retaining wall of entrance to tunnel in Zhong state [3]. Cai Yang researched reliability design method of the gravity type retaining wall, comparatively systematically, and discussed to the determination of dividing coefficient in the terminal state design of probability [4]. Du Yong-Feng regarded overturning and sliding of retaining wall as connecting system, calculating the reliability index of the structural 
system in the progressively equivalent plane method. The structural system failure probability of the retaining wall stability was computed by the reliability index of the structural system finally [5]. Considering unstable retaining wall randomness and fuzzy synthetically wholly, Wang Guang-Yue set up basic analyzing model of retaining wall fuzzy reliability and proposes the computing technology of fuzzy[6]. However, the first five literatures only considered random uncertainty during analyzing retaining wall stability. Though Wang Guang-Yue considered the fuzzy uncertainty during the analysis of retaining wall's stability, supposed $R, S$ and structural performance function $\mathrm{Z}$ to obey normal distribution, thus, obtained the density function $\mathrm{f}(\mathrm{Z})$ of probability, and then calculated the random fuzzy reliability in the integral method. However, the establishment of this assumption remains to be proved and the rationality of this treatment is worth discussing. This text gets around the assumption, adopting cut-set method to turn fuzzy set into regular interval set, seeking fuzzy random reliability through inter-zone value. Thus the result of calculation is more reasonable.

\section{Analyzing Model in Fuzzy Dependability}

The traditional dependability theory describes the demarcation line between reliable and invalid structure with the terminal state, and uses the terminal equation $\mathrm{Z}=\mathrm{R}-\mathrm{S}=0$, as Fig.1 (a) shows, to express. However, it is very difficult to define demarcation line between the reliable and invalid, because there exists a transition state during that time and that is a fuzzy range which shows that efficiency is losing gradually, seen Fig.1 (b). The equation for the fuzzy performance function is

$$
\tilde{Z}=\tilde{R}-\tilde{S}
$$

While fuzziness exists, the safe state should be regarded as a fuzzy incident $\tilde{A}$, whose subjection degree can be described by the performance function $\mathrm{Z}$. namely

$$
\mu_{\lambda}(Z)=\left\{\begin{array}{l}
1, \text { The component is in safe state } \\
\in(0,1), \text { The component is in fuzzy terminal state } \\
0, \text { The component is in invalid state }
\end{array}\right.
$$

So, fuzzy reliability refers to the fuzzy probability of incident $\tilde{A}$, and namely the probability of $\mathrm{Z}=\mathrm{g}(\mathrm{x}) \stackrel{\sim}{\geq}$, it is expressed as

$$
P_{r}=p(z \stackrel{\sim}{\geq} 0)
$$

So, the structure fuzzy random reliability formula is

$$
p_{r}=p(\tilde{A})=\int_{-\infty}^{+\infty} \mu_{\lambda}(x) f(x) d x
$$

Among (10) formula: $f(x)$ is distribution density function of basic random variable, and $\mu_{\lambda}(x)$ is subjection function of fuzzy safe incident. Two special cases are:

(1)When most great load effect and resist strength of structure have only randomness without fuzziness, its subjection degree is one in the valid intra-area, and is zero in the valid extra-area. Then the formula (10) is turned into

$$
P_{r}=\int_{\Omega} f(x) d x
$$

Formula (11) is a regular reliability calculating type.

(2)When most great load effect and resist strength of structure have only fuzziness without randomness, $\mu_{\lambda}(x)$ will turn into a constant $u_{\lambda}$, and formula (10) will turn into $\operatorname{Pr}=u_{\lambda}$, which is 
subjection degree of structure reaction for a incident of structure safety and is called ordinary fuzzy reliability [8].It indicates that when structural parameters have only fuzziness, the structure will produce dependability problem too.

\section{Fuzzy Reliability Calculation Model of Gravity Retaining Wall}

There are two ways in fuzzy reliability calculation. First, fuzzy random phenomenon is described by the fuzzy set, and the fuzzy reliability of the structure is defined to utilize fuzzy incident probability. Then it is calculated directly through the formula (10) by total mark way. Second, the fuzzy random phenomenon is described by fuzzy random variable which is regarded as basic variable. Structure reliability is calculated through $\lambda$ level cut set method [9]. It is very miscellaneous to calculate by total mark method which is unable to solve often, because it difficult to receive concrete expression formula of distribution density function and subjection function of fuzzy incident. Fuzzy random reliability is solved to adopt cut set way which turns fuzzy set into regular interval set and receiving inter-zone solution.

\section{The Setting-up of Gravity Retaining Wall Structural Performance Function}

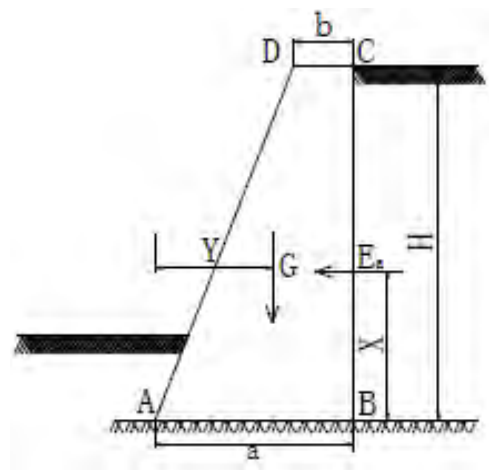

Fig. 3 Gravity Retaining Wall

The major unstable modes of gravity retaining walls are overturning and sliding. As fig.2 shows, resisting overturning steady performance function which rotates wall toe is

$$
\mathrm{Z} 1=g_{1}\left(M_{R}, M_{S}\right)=M_{R}-M_{S}=G Y-\frac{1}{2} r\left(H-\frac{2 c}{r \sqrt{K_{a}}}\right)^{2} K_{a} \bullet X
$$

Resisting sliding steady performance function is

$$
\begin{aligned}
& \mathrm{Z} 2=g_{2}\left(F_{R}, F_{S}\right)=F_{R}-F_{S}=G \mu-\frac{1}{2} r\left(H-\frac{2 c}{r \sqrt{K_{a}}}\right)^{2} K_{a} \\
& K_{a}=\tan ^{2}\left(45^{0}-\frac{\varphi}{2}\right)
\end{aligned}
$$

In formula (12) and (13), G-stands for the deadweight of retaining wall each extending meter; r-stands for the soil weight degree behind wall; $\mathrm{H}$-stands for the height of retaining wall; c-stands for the gluing strength of soil body behind wall; Ka-stands for the initiative soil pressure coefficient; $\mathrm{X}$ and $\mathrm{Y}$ are arms-to- to toe of wall weight strength and initiative soil pressure respectively; $\mu$-stands for soil to basal retaining wall friction coefficient; $\varphi$-stands for the Interior friction angle of soil body behind wall. [10]

As the gravity retaining wall illustrated in fig.2, neglecting wall peak load and passive soil pressure of retaining wall, the geometric size of the retaining wall and physical parameter are known quantity. A large number of project practices prove that main fuzzy random variables that influences retaining walls are $\tilde{r}, \tilde{c}, \tilde{\varphi}, \tilde{\mu}$, which these four fuzzy random variables are normal 
distribution usually. [11] In order to express conveniently, the above four fuzzy random variables are replaced separately by $\tilde{x}_{i}(i=1,2,3,4)$.under the situations that the fuzzy mean value $\tilde{m}_{x i}$ and fuzzy standard deviation $\tilde{\sigma}_{x i}$ of statistical parameters are known, $\tilde{\beta}_{1}$ and $\tilde{\beta}_{2}$ of fuzzy dependability indexes can be solved following the analytic approach of structural fuzzy reliability.[12]

\section{The Fuzzy Reliability Calculation of Gravity Retaining Walls}

The equation of fuzzy random terminal state can be set up

$$
\tilde{Z}=\tilde{F}-1=\tilde{R}-\tilde{S}=g\left(\tilde{x}_{i}\right)=0 \quad(\mathrm{i}=1,2,3,4)
$$

On the basis of first - order second - moment method, Taylor progression is adopted to outspread terminal state functions in central points, and make its linearization

$$
Z \approx g\left(\tilde{m}_{x 1}, \tilde{m}_{x 2}, \tilde{m}_{x 3}, \tilde{m}_{x 4}\right)+\sum_{n=1}^{i}\left(\tilde{x}_{n}-\tilde{m}_{x n}\right) \frac{\partial g}{\partial x_{n}}
$$

Then, fuzzy dependability index $\tilde{\beta}$ can be expressed as

$$
\tilde{\beta}=\frac{\tilde{m}_{z}}{\tilde{\sigma}_{z}}
$$

Among (24) formula [13]

$$
\left\{\begin{array}{l}
\tilde{m}_{z} \approx g\left(\tilde{m}_{x 1}, \tilde{m}_{x 2}, \tilde{m}_{x 3}, \tilde{m}_{x 4}\right) \\
\tilde{\sigma}_{z}^{2} \approx \sum_{n=1}^{k} \tilde{\sigma_{x n}^{2}}\left(\frac{\partial g}{x_{n}} \mid \tilde{m}_{x n}\right)^{2}
\end{array}\right.
$$

Under $\lambda$ level cut set, the dependability index value is

$$
\left\{\begin{array}{l}
\tilde{\beta}_{\lambda}=\left[\beta_{\lambda}^{l}, \beta_{\lambda}^{l}\right]=\frac{m_{z \lambda}}{\tilde{\sigma}_{z \lambda}}=\left[\left(\frac{m_{z}}{\sigma_{z}}\right)_{\lambda}^{l},\left(\frac{m_{z}}{\sigma_{z}}\right)_{\lambda}^{u}\right] \\
\left(\frac{m_{z}}{\sigma_{z}}\right)_{\lambda}^{l}=\min \frac{g\left(\tilde{m}_{x 1}, \tilde{m}_{x 2}, \tilde{m}_{x 3}, \tilde{m}_{x 4}\right)}{\sum_{m=1}^{4} \sigma_{x n}^{2}\left(\frac{\partial g}{\partial x_{m}} \mid m_{x m}\right)^{2}} \\
\left(\frac{m_{z}}{\sigma_{z}}\right)_{\lambda}^{\mu}=\max \frac{g\left(\tilde{m}_{x 1}, \tilde{m}_{x 2}, \tilde{m}_{x 3}, \tilde{m}_{x 4}\right)}{\sum_{m=1}^{4} \sigma_{x n}^{2}\left(\frac{\partial g}{\partial x_{m}} \mid m_{x m}\right)^{2}}
\end{array}\right.
$$

Among (26) formula, $\beta_{\lambda}^{u}$ and $\beta_{\lambda}^{l}$ express up and down bounds of dependability fuzzy index $\tilde{\beta}$ in $\lambda$ level cut set respectively $\tilde{m}_{x n}$ and $\tilde{\sigma}_{x n}^{2}$ separately express the inter-zone of the nth fuzzy mean value and fuzzy square variance under the condition of $\lambda$ cut set. Max and min respectively express that fetch upper and low bound value of inter-zone numbers. According to the inter-zone reliability calculation $[14,15]$, under $\lambda$ level cut set, structural fuzzy reliability value is

$$
p_{r \lambda}=\frac{1}{\beta^{u}-\beta^{l}} \int_{\beta^{l}}^{\beta^{u}} \phi(\beta) d \beta=E[\phi(\beta)]
$$

After carrying on total mark of change order of the right of formula (27), the answer is 


$$
\int_{\beta^{l}}^{\beta^{u}} \phi(\beta) d \beta \int_{\beta^{l}}^{\beta^{u}}\left[\int_{-\infty}^{\beta} \frac{1}{\sqrt{2 \pi}} \exp \left(-\frac{t^{2}}{2}\right) d t\right] d \beta=\beta^{u} \phi\left(\beta^{u}\right)-\beta^{l} \phi\left(\beta^{l}\right)+\frac{1}{\sqrt{2 \pi}}\left\{\exp \left[-\frac{\left(\beta^{u}\right)^{2}}{2}\right]-\exp \left(-\frac{\left(\beta^{l}\right)^{2}}{2}\right)\right\}
$$

So, the estimated value of the fuzzy reliability is

$$
p_{r}=\int_{0}^{l} p_{r \lambda} d \lambda
$$

It can also be approximately expressed as

$$
p_{r} \approx \frac{1}{n} \sum_{i=1}^{n} p_{r \lambda i}
$$

\section{The Calculation Example}

A certain gravity retaining wall as shown in fig.2, it has straight and smooth back, and is neglecting the passive soil pressure. There is not added loading on the top of the retaining wall. The structural parameter of the wall body is invariable, among them: $\mathrm{H}=5.4 \mathrm{~m}, \mathrm{X}=1.14 \mathrm{~m}, \mathrm{G}=98.8 \mathrm{KN} / \mathrm{m}$, $\mathrm{Y}=1.62 \mathrm{~m} . \mathrm{a}=1.0 \mathrm{~m}, \mathrm{~b}=3.6 \mathrm{~m}$. Subjection function of every $\mathrm{R}-\mathrm{L}$ type fuzzy numbers is a linear one. $\tilde{r}, \tilde{c}, \tilde{\varphi}$ and $\tilde{\mu}$ are the fuzzy random variable. $\tilde{r}=((21,2.2,3.2) \mathrm{LR},(0.1,0.01,0.015) \mathrm{LR}), \tilde{c}=$ $((12,1.5,2.1) \mathrm{LR},(0.1,0.01,0.015) \mathrm{LR}), \tilde{\varphi}=\left(\left(40^{\circ}, 4^{\circ}, 4.2^{\circ}\right) \mathrm{LR},(0.1,0.01,0.015) \mathrm{LR}\right), \tilde{\mu}=((0.5$, $0.01,0.03) \operatorname{LR}(0.1,0.01,0.015) \mathrm{LR})$.

The result of calculation is shown as table 1.It can be found out from table 1: fuzziness neglected, the result will be more deviation. While fuzziness exists objectively, so, it is more reasonable if only fuzziness is considered. Meanwhile, it can be found that fuzzy dependability index and fuzzy reliability in the first - order second - moment method, is less than the values without considering fuzziness. So, using the fuzzy dependability analyzing model weights the dependability is even more secure.

Tab. 1 Comparison of Calculated Results

\begin{tabular}{lcccc}
\hline \multicolumn{1}{c}{ Model types } & \multicolumn{2}{c}{$\begin{array}{c}\text { Reliability index } \\
\text { overturning }\left(\beta_{1}\right)\end{array}$} & sliding $\left(\beta_{2}\right)$ & \multicolumn{2}{c}{$\begin{array}{c}\text { Reliability } \\
\text { overturning }\left(P_{\mathrm{r} 1}\right)\end{array}$} & sliding $\left(\mathrm{P}_{\mathrm{n} 2}\right)$ \\
\hline $\begin{array}{l}\text { classical dependability } \\
\text { analyzing model }\end{array}$ & 2.2345 & 2.0243 & 0.98501 & 0.97805 \\
$\begin{array}{l}\text { Literature [6]fuzzy depend- } \\
\text { ability analyzing model }\end{array}$ & 1.9601 & 1.9101 & 0.97395 & 0.97483 \\
$\begin{array}{l}\text { Analyzing model fuzzy } \\
\text { dependability of this text }\end{array}$ & 1.8066 & 1.7887 & 0.960065 & 0.95862 \\
\hline
\end{tabular}

\section{Conclusion}

(1)Combining the theory of random reliability with fuzzy mathematics, the fuzzy random reliability analyzing model of the gravity retaining wall's structure is set up. Through the decomposing theorem, the fuzzy variable was changed into a series of interval variable operation, overturning and sliding reliability indexes are received, and their reliabilities are also obtained respectively. This kind of computing technology can be regarded as the new development of the structural design of gravity retaining wall. The fuzzy analysis theory and computing technology of reliability that this text puts forward, have certain directive significance to other project structural design too.

(2)The study shows that the calculation amount of this model and the one classical reliability analyzing model are roughly equal. The Concrete calculation example proves there are certain differences between fuzzy reliability model and classical model. So, when analyzing reliability, fuzziness cannot be neglected. 


\section{Acknowledgement}

Fund project: The national natural science fund (51308424); Plan of action about young College Teachers Participating in business in Hubei Province(005070); High-speed railway construction of National Engineering Laboratory in Central South University(HSR2013006).

Author's profile: XIAO Zunqun(1982-), male, Chinese, han, from Huaihua of Hunan, doctor and Communication people, engage in rock soil, heavily loaded railway research work mainly, Tel: 18831646185,E-mail: xiaozunqun@126.com..

\section{References}

[1]ENRQUE C, ROBERTO M, ANA R T, ALFONSO F C. Design and sensitivity analysis using the probability-safety-factor method: an application to retaining walls[J]. Structural Safety, 2004, 26(2):159-179.

[2]ZHANG Jian-ren. Reliability analysis of stability for retaining wall structure [J].China Journal of Highway and Transport, 1997, 10(3): 53-58. (In Chinese)

[3]WANG Liang, LIU Yuan-xue. Reliability analysis of gravity retaining wall stability against slide [J].Journal of Chongqing Technology and Business University (Natural Science), 2005, 22(6): 609-611. (In Chinese)

[4]CAI Yang.Research on reliability of gravity retaining wall [D].Chengdu: Southwest Jiaotong University, 2005. (In Chinese)

[5]DU Yong-feng. Analysis of reliability of structural systems for stability of gravity retaining walls [J].Chinese Journal of Geotechnical Engineering, 2008, 30(3):627-631. (In Chinese)

[6]WANG Guang-yue. Analysis of fuzzy reliability for retaining wall stability [J].Journal of Shan-Dong university (engineering science), 2003, 33(3):627-641. (In Chinese)

[7]DUB0IS D, PRADE H.Fuzzy Sets and System:Theory and Applications[M].New York: Academic Press, 1980.

[8]JIANQM,CHENCH.An numerical algorithm of fuzzy reliability [J].Reliability Engineering and System Safety, 2003, 80(3): 299-307.

[9] HAN Jing.Fuzzy Stochastic Reliability Analysis of Engineering Structure [J].2008, 7(3):372-373. (In Chinese)

[10]HASFER A M, LIND N C. Exact and invariant second-moment code format[J].Engineering Mechanics ASCE, 1974, 100(1):111-121.

[11]CHANG T Y, TANIGUCHI H, CHEN W F. Nonlinear finite element analysis of reinforced concrete panels [J]. Journal of Structure Engineering, 1987, 113(1):122-140.

[12]CHEN S M.Analyzing fuzzy system reliability using vague set theory[J].International Journal of Applied Scienceand Engineering, 2003, 1(1):82-88.

[13]DODAGOUDAR G R, VENKATACHALAM G.Reliability analysis of slopes using fuzzy sets theory[J].Computers and Geotechnics, 2000, (27)3:10-1 15.

[14]LV Xi-ling,LV En-ling. Strnctural system fuzzy reliability analysis [J].Journal of Chongqing University:Natural Science Edition, 2 006.29(7):119-123.(In Chinese)

[15]GUO Shu-xiang, LV Zhen-zhou. A non-probabilistic model of structural reliability based on interval analysis [J]. (Chinese Journal of Computational Mechanics, 2001, 18(1):56-60. (In Chinese) 